\title{
ON RIGIDITY OF GRADIENT KÄHLER-RICCI SOLITONS WITH HARMONIC BOCHNER TENSOR
}

\author{
QIANG CHEN AND MENG ZHU
}

(Communicated by Lei $\mathrm{Ni}$ )

\begin{abstract}
In this paper, we prove that complete gradient steady KählerRicci solitons with harmonic Bochner tensor are necessarily Kähler-Ricci flat, i.e., Calabi-Yau, and that complete gradient shrinking (or expanding) KählerRicci solitons with harmonic Bochner tensor must be isometric to a quotient of $N^{k} \times \mathbb{C}^{n-k}$, where $N$ is a Kähler-Einstein manifold with positive (or negative) scalar curvature.
\end{abstract}

\section{INTRODUCTION}

A complete Riemannian manifold $\left(M, g_{i j}\right)$ is called a Ricci soliton if there is a vector field $X$ and a constant $\lambda$ such that

$$
R_{i j}+\frac{1}{2}\left(\nabla_{i} X_{j}+\nabla_{j} X_{i}\right)=\frac{\lambda}{2} g_{i j}
$$

where $\lambda>0, \lambda=0$, or $\lambda<0$ corresponds to a shrinking, steady or expanding soliton respectively. Moreover, a Ricci soliton is called a gradient Ricci soliton if the vector field is a gradient vector field, i.e. $X=\nabla f$ for some smooth function $f$ on $M$. In this case, the Ricci soliton equation above becomes

$$
R_{i j}+\nabla_{i} \nabla_{j} f=\frac{\lambda}{2} g_{i j}
$$

Since R. Hamilton [18 introduced the concept of Ricci solitons in the mid 1980's, the study of Ricci solitons has attracted a lot of attention. Ricci solitons, as selfsimilar solutions to Hamilton's Ricci flow, are natural generalizations of Einstein metrics. Since they often arise as the dilation limits of the singularities of the Ricci flow, Ricci solitons play an important role in the singularity analysis of the Ricci flow.

In the past decade, a significant number of results have been obtained in classifying or understanding the geometry of shrinking solitons. In particular, the classification of gradient shrinkers is known in dimensions 2 and 3, and assuming locally conformally flatness in all dimensions $n \geq 4$; see, e.g., [23], 22], 7], [16, 25], [27], 12], 21], 20], etc. Recently, a rigidity result for Bach flat gradient shrinkers has also been obtained by H.-D. Cao and the first author [9]. We refer the reader to a recent survey paper of H.-D. Cao [5] on Ricci shrinkers.

Regarding steady solitons, it is well-known that compact ones (as well as compact expanding solitons) must be Einstein; see, e.g., [11 for a proof. However, much less

Received by the editors May 12, 2011.

2010 Mathematics Subject Classification. Primary 53C44, 53C55.

Key words and phrases. Kähler-Ricci soliton, Bochner tensor, rigidity. 
is known for noncompact steady Ricci solitons. In dimension $n=2$, R. Hamilton showed that any 2-dimensional steady soliton is isometric to the Cigar soliton, up to scaling. For $n=3$, G. Perelman [23] conjectured that any 3-dimensional complete ( $\kappa$-noncollapsed) steady soliton with positive sectional curvature is isometric to the Bryant soliton, which is the unique rotationally symmetric example on $\mathbb{R}^{3}$. Very recently, H.-D. Cao and the first author 8 made the first important progress on this problem. They showed that any nonflat $n$-dimensional, $n \geq 3$, complete locally conformally flat steady Ricci soliton is isometric to the Bryant soliton (for $n \geq 4$, Catino and Mantegazza [13] independently proved this result by using a different method). Subsequently, their work has been used by S. Brendle 1 in classifying 3-dimensional steady Ricci solitons satisfying certain asymptotic conditions, and $\mathrm{X}$. X. Chen and Y. Wang 15] in classifying 4-dimensional half-conformally flat steady solitons, respectively. Most recently, H.-D. Cao, G. Catino, C. Mantegazza, L. Mazzieri and the first author [6] have proved that complete Bach-flat gradient steady solitons with positive Ricci curvature such that the scalar curvature attains a maximum at an interior point are also isometric to the Bryant soliton.

For more thorough discussions and results in Ricci solitons, the reader can look at the survey [4] of H.-D. Cao and the references therein. From now on, we will focus on Kähler-Ricci solitons.

Definition 1.1. An $n$-dimensional Kähler manifold $\left(M^{n}, g_{i}\right)$ is called a gradient Kähler-Ricci soliton if there is a real-valued smooth function $f$ satisfying the soliton equation

$$
R_{i \bar{j}}+\nabla_{i} \nabla_{\bar{j}} f=\lambda g_{i \bar{j}},
$$

for some constant $\lambda \in \mathbb{R}$ and such that $\nabla f$ is a holomorphic vector field, i.e. $\nabla_{i} \nabla_{j} f=0$.

In [10, H.-D. Cao and R. Hamilton observed that complete noncompact gradient steady Kähler-Ricci solitons with positive Ricci curvature such that the scalar curvature attains the maximum must be Stein (and diffeomorphic to $\mathbb{R}^{2 n}$ ). Later, under the same assumptions, A. Chau and L.-F. Tam [14, and R. Bryant [3] independently proved that such steady Kähler-Ricci solitons are actually biholomorphic to $\mathbb{C}^{n}$. Moreover, Chau and Tam showed that complete noncompact expanding Kähler-Ricci solitons with nonnegative Ricci curvature are also biholomorphic to $\mathbb{C}^{n}$.

To state our results, let us first recall that on Kähler manifolds there is a similar notion to the Weyl tensor, called the Bochner tensor. The Bochner tensor $W_{i \bar{j} k \bar{l}}$ is defined by

$$
\begin{aligned}
W_{i \bar{j} k \bar{l}}= & R_{i \bar{j} k \bar{l}}-\frac{R}{(n+1)(n+2)}\left(g_{i \bar{j}} g_{k \bar{l}}+g_{i \bar{l}} g_{k \bar{j}}\right) \\
& +\frac{1}{(n+2)}\left(R_{i \bar{j}} g_{k \bar{l}}+R_{k \bar{l}} g_{i \bar{j}}+R_{i \bar{l}} g_{k \bar{j}}+R_{k \bar{j}} g_{i \bar{l}}\right),
\end{aligned}
$$

where $R_{i \bar{j}}=g^{k \bar{l}} R_{i \bar{j} k \bar{l}}$. We also denote the divergence of the Bochner tensor by

$$
\begin{aligned}
C_{i \bar{j} k} & =g^{l \bar{q}} \nabla_{l} W_{i \bar{j} k \bar{q}} \\
& =\frac{n}{n+2} \nabla_{i} R_{k \bar{j}}-\frac{n}{(n+1)(n+2)}\left(g_{k \bar{j}} \nabla_{i} R+g_{i \bar{j}} \nabla_{k} R\right) .
\end{aligned}
$$


Definition 1.2. A Kähler manifold $M^{n}$ is said to have harmonic Bochner tensor if $C_{i \bar{j} k}=0$, i.e.,

$$
\nabla_{i} R_{k \bar{j}}=\frac{1}{n+1}\left(g_{k \bar{j}} \nabla_{i} R+g_{i \bar{j}} \nabla_{k} R\right)
$$

Very recently, by using a similar argument as in the paper 8] of H.-D. Cao and the first author, Y. Su and K. Zhang 26] have shown that any complete noncompact gradient Kähler-Ricci soliton with vanishing Bochner tensor is necessarily KählerEinstein, and hence a quotient of the corresponding complex space form.

In this paper we investigate gradient Kähler-Ricci solitons with harmonic Bochner tensor, and extend the classification results of $\mathrm{Su}$ and Zhang. Our main results are:

Theorem 1.3. Any complete gradient steady Kähler-Ricci soliton with harmonic Bochner tensor must be Kähler-Ricci flat (i.e., Calabi-Yau).

Theorem 1.4. Any complete gradient shrinking (or expanding) Kähler-Ricci soliton with harmonic Bochner tensor must be isometric to the quotient of $N^{k} \times \mathbb{C}^{n-k}$, where $N^{k}$ is a $k$-dimensional Kähler-Einstein manifold with positive (or negative) scalar curvature.

Remark 1.5. It is known that a compact Kähler manifold with vanishing Bochner tensor (also called Bochner-Kähler or Bochner-flat) is necessarily a compact quotient of $M_{c}^{k} \times M_{-c}^{n-k}$, where $M_{c}^{k}$ and $M_{-c}^{n-k}$ denote the complex space forms of constant holomorphic sectional curvature $c$ and $-c$ respectively (cf., e.g., Corollary 4.17 in [2]). It follows immediately that any compact Kähler-Ricci soliton with vanishing Bochner tensor must be a quotient of a complex space form.

Remark 1.6. In the Riemannian case, by using a rigidity result of Petersen and Wylie [24, Fernández-López and García-Río [17, and Munteanu and Sesum [20] proved that Ricci shrinkers with harmonic Weyl tensor must be rigid, i.e., a quotient of the product of an Einstein manifold and $\mathbb{R}^{k}$.

\section{Proof of the MAIN THEOREMS}

Let $\left(M^{n}, g_{i \bar{j}}, f\right)$ be a gradient Kähler-Ricci soliton, i.e.

$$
R_{i \bar{j}}+\nabla_{i} \nabla_{\bar{j}} f=\lambda g_{i \bar{j}} \quad \text { and } \quad \nabla_{i} \nabla_{j} f=0 .
$$

It is well-known that the following basic identities hold (see e.g. [11]).

Lemma 2.1. On a gradient Kähler-Ricci soliton, we have

$$
\begin{gathered}
R+|\nabla f|^{2}-\lambda f=\text { Const, } \\
R+\Delta f=n \lambda, \\
\nabla_{i} R_{k \bar{j}}=R_{i \bar{j} k \bar{l}} \nabla_{l} f,
\end{gathered}
$$

and

$$
\nabla_{i} R=R_{i \bar{j}} \nabla_{j} f
$$

From now on, we assume that $\left(M^{n}, g_{i \bar{j}}, f\right)$ is a gradient Kähler-Ricci soliton with harmonic Bochner tensor so that

$$
\nabla_{i} R_{k \bar{j}}=\frac{1}{n+1}\left(\nabla_{i} R g_{k \bar{j}}+\nabla_{k} R g_{i \bar{j}}\right)
$$


Lemma 2.2. We have

$$
\begin{aligned}
& \lambda R_{i \bar{j}}-R_{i \bar{j} k \bar{l}} R_{\bar{k} l} \\
& \begin{array}{l}
\frac{1}{n+1}[ \\
\quad \frac{1}{n+1} \nabla_{k} R \nabla_{\bar{k}} f g_{i \bar{j}}+\left(\lambda R-|R c|^{2}\right) g_{i \bar{j}}-\frac{n}{n+1} \nabla_{i} R \nabla_{\bar{j}} f \\
\left.\quad+\lambda R_{i \bar{j}}-R_{i \bar{k}} R_{k \bar{j}}\right]
\end{array}
\end{aligned}
$$

and

$$
\begin{aligned}
& 2(n+1) \lambda \nabla_{i} R-2 R \nabla_{i} R-2 R_{i \bar{j}} \nabla_{j} R \\
& =-\frac{1}{n+1} \nabla_{i} R|\nabla f|^{2}-\frac{1}{n+1} \nabla_{k} R \nabla_{\bar{k}} f \nabla_{i} f .
\end{aligned}
$$

Proof. On the one hand, by differentiating (2.4), we obtain

$$
\Delta R=\nabla_{k} \nabla_{\bar{k}} R=\nabla_{k} R \nabla_{\bar{k}} f+R_{k \bar{l}} \nabla_{\bar{k}} \nabla_{l} f .
$$

From (2.5), we get

$$
\begin{aligned}
\nabla_{k} \nabla_{\bar{k}} R_{i \bar{j}}= & \frac{1}{n+1}\left(\Delta R g_{i \bar{j}}+\nabla_{i} \nabla_{\bar{j}} R\right) \\
= & \frac{1}{n+1}\left(\nabla_{k} R \nabla_{\bar{k}} f g_{i \bar{j}}+R_{k \bar{l}} \nabla_{\bar{k}} \nabla_{l} f g_{i \bar{j}}+\nabla_{i} R_{k \bar{j}} \nabla_{\bar{k}} f+R_{k \bar{j}} \nabla_{i} \nabla_{\bar{k}} f\right) \\
= & \frac{1}{n+1}\left[\nabla_{k} R \nabla_{\bar{k}} f g_{i \bar{j}}+\left(\lambda R-|R c|^{2}\right) g_{i \bar{j}}+\frac{1}{n+1} \nabla_{i} R \nabla_{\bar{j}} f\right. \\
& \left.\quad+\frac{1}{n+1} \nabla_{k} R \nabla_{\bar{k}} f g_{i \bar{j}}+\lambda R_{i \bar{j}}-R_{i \bar{k}} R_{k \bar{j}}\right] .
\end{aligned}
$$

On the other hand, by differentiating (2.3), we have

$$
\begin{aligned}
\nabla_{k} \nabla_{\bar{k}} R_{i \bar{j}} & =\nabla_{i} R_{\bar{j} l} \nabla_{\bar{l}} f+R_{i \bar{j} k \bar{l}} \nabla_{\bar{k}} \nabla_{l} f \\
& =\nabla_{k} R_{i \bar{j}} \nabla_{\bar{k}} f+R_{i \bar{j} k \bar{l}} \nabla_{\bar{k}} \nabla_{l} f \\
& =\nabla_{k} R_{i \bar{j}} \nabla_{\bar{k}} f+\lambda R_{i \bar{j}}-R_{i \bar{j} k \bar{l}} R_{\bar{k} l} .
\end{aligned}
$$

Now, by plugging in formula (2.8), we obtain (2.6).

Next, by taking the divergence on both sides of (2.6), we get

$$
\begin{aligned}
& \lambda \nabla_{i} R-\left(\nabla_{i} R_{k \bar{l}}\right) R_{\bar{k} l}-R_{i \bar{j} k \bar{l}} \nabla_{j} R_{\bar{k} l} \\
& =\frac{1}{n+1}\left[\frac{1}{n+1} \nabla_{i} \nabla_{k} R \nabla_{\bar{k}} f+\frac{1}{n+1} \nabla_{k} R \nabla_{i} \nabla_{\bar{k}} f+\lambda \nabla_{i} R-\nabla_{i}|R c|^{2}\right. \\
& \left.-\frac{n}{n+1} \nabla_{j} \nabla_{i} R \nabla_{\bar{j}} f-\frac{n}{n+1} \nabla_{i} R \Delta f+\lambda \nabla_{i} R-\left(\nabla_{j} R_{i \bar{k}}\right) R_{k \bar{j}}-R_{i \bar{k}} \nabla_{k} R\right] \\
& =\frac{1}{n+1}\left[\frac{1}{n+1} \nabla_{i} R_{k \bar{l}} \nabla_{l} f \nabla_{\bar{k}} f+\frac{\lambda}{n+1} \nabla_{i} R-\frac{1}{n+1} R_{i \bar{k}} \nabla_{k} R+\lambda \nabla_{i} R-2 R_{k \bar{l}} \nabla_{i} R_{\bar{k} l}\right. \\
& -\frac{n}{n+1} \nabla_{i} R_{j \bar{k}} \nabla_{k} f \nabla_{\bar{j}} f-\frac{\lambda n^{2}}{n+1} \nabla_{i} R+\frac{n}{n+1} R \nabla_{i} R+\lambda \nabla_{i} R \\
& \left.-R_{k \bar{j}} \nabla_{i} R_{j \bar{k}}-R_{i \bar{k}} \nabla_{k} R\right] \text {. }
\end{aligned}
$$


That is,

$$
\begin{aligned}
& \lambda \nabla_{i} R-\left(\nabla_{i} R_{k \bar{l}}\right) R_{\bar{k} l}-R_{i \bar{j} k \bar{l}} \nabla_{j} R_{\bar{k} l} \\
& =\frac{1}{n+1}\left[-\frac{n-1}{(n+1)^{2}} \nabla_{i} R|\nabla f|^{2}-\frac{n-1}{(n+1)^{2}} \nabla_{k} R \nabla_{\bar{k}} f \nabla_{i} f\right. \\
& \left.\quad+(3-n) \lambda \nabla_{i} R-\left(1+\frac{1}{n+1}\right) R_{i \bar{k}} \nabla_{k} R-3 R_{k \bar{l}} \nabla_{i} R_{\bar{k} l}+\frac{n}{n+1} R \nabla_{i} R\right] .
\end{aligned}
$$

But,

$$
\begin{aligned}
R_{l \bar{k}} \nabla_{i} R_{k \bar{l}} & =\frac{1}{n+1} R_{l \bar{k}}\left(\nabla_{i} R g_{k \bar{l}}+\nabla_{k} R g_{i \bar{l}}\right) \\
& =\frac{1}{n+1} R \nabla_{i} R+\frac{1}{n+1} R_{i \bar{j}} \nabla_{j} R
\end{aligned}
$$

and

$$
\begin{aligned}
R_{i \bar{j} k \bar{l}} \nabla_{j} R_{l \bar{k}} & =\frac{1}{n+1} R_{i \bar{j} k \bar{l}}\left(\nabla_{j} R g_{l \bar{k}}+\nabla_{l} R g_{j \bar{k}}\right) \\
& =\frac{1}{n+1} R_{i \bar{j}} \nabla_{j} R+\frac{1}{n+1} R_{i \bar{l}} \nabla_{l} R \\
& =\frac{2}{n+1} R_{i \bar{j}} \nabla_{j} R .
\end{aligned}
$$

Hence, we have

$$
\begin{aligned}
& \lambda \nabla_{i} R-\frac{1}{n+1} R \nabla_{i} R-\frac{3}{n+1} R_{i \bar{j}} \nabla_{j} R \\
& =\lambda \nabla_{i} R-\left(\nabla_{i} R_{k \bar{l}}\right) R_{\bar{k} l}-R_{i \bar{j} k \bar{l}} \nabla_{j} R_{\bar{k} l} \\
& =\frac{1}{n+1}\left[-\frac{n-1}{(n+1)^{2}} \nabla_{i} R|\nabla f|^{2}-\frac{n-1}{(n+1)^{2}} \nabla_{k} R \nabla_{\bar{k}} f \nabla_{i} f\right. \\
& \left.\quad \quad+(3-n) \lambda \nabla_{i} R-\left(1+\frac{1}{n+1}\right) R_{i \bar{k}} \nabla_{k} R-3 R_{k \bar{l}} \nabla_{i} R_{\bar{k} l}+\frac{n}{n+1} R \nabla_{i} R\right] .
\end{aligned}
$$

Therefore, formula (2.7) follows easily.

Now, suppose that $\nabla f \neq 0$ at some point $p$. Then we may choose an orthonormal frame $\left\{e_{1}, e_{2}, \cdots, e_{n}\right\}$ of holomorphic vector fields at $p$ such that $e_{1}$ is parallel to $\nabla f$. Therefore, we have $\left|\nabla_{1} f\right|=|\nabla f|$ and $\nabla_{k} f=0$ for $k=2, \cdots, n$.

Lemma 2.3. Suppose $\nabla f \neq 0$ at $p$. Then, under the frame $\left\{e_{1}, e_{2}, \cdots, e_{n}\right\}$ chosen above, we have

$$
R_{k \overline{1}}=R_{1 \bar{k}}=0 \quad \text { for } \quad k \geq 2 .
$$

Proof. From (2.3) and (2.5), we have at $p$,

$$
R_{i \bar{j} k \overline{1}} \nabla_{1} f=\frac{1}{n+1}\left(\nabla_{i} R g_{k \bar{j}}+\nabla_{k} R g_{i \bar{j}}\right)=\frac{1}{n+1}\left(R_{i \overline{1}} g_{k \bar{j}}+R_{k \overline{1}} g_{i \bar{j}}\right) \nabla_{1} f
$$

It follows that

$$
R_{i \bar{j} k \overline{1}}=\frac{1}{n+1}\left(R_{i \overline{1}} g_{k \bar{j}}+R_{k \overline{1}} g_{i \bar{j}}\right) .
$$

In particular, for $k \geq 2$, we have that

$$
R_{1 \overline{1} k \overline{1}}=\frac{1}{n+1} R_{k \overline{1}} \quad \text { and } \quad R_{1 \bar{k} 1 \overline{1}}=0 .
$$


However, on the other hand, it is easy to see that

$$
R_{1 \overline{1} k \overline{1}}=\overline{R_{\overline{1} 1 \bar{k} 1}}=\overline{R_{1 \bar{k} 1 \overline{1}}}=0 .
$$

Therefore, $R_{k \overline{1}}=R_{1 \bar{k}}=0$ for $k \geq 2$.

Lemma 2.3 tells us that $\nabla f$ is an eigenvector of the Ricci curvature tensor. Thus we may choose another orthonormal frame $\left\{w_{1}=e_{1}, w_{2}, \cdots, w_{n}\right\}$ at $p$ such that $\left|\nabla_{1} f\right|=|\nabla f|$ and the Ricci curvature tensor is diagonalized at $p$, i.e.

$$
R_{i \bar{j}}=R_{i \bar{i}} \delta_{i j} .
$$

Proposition 2.4. Suppose that $\nabla f \neq 0$ at $p$. Then under the orthonormal frame $\left\{w_{1}, w_{2}, \cdots, w_{n}\right\}$ chosen above, we have the following identities at $p$ :

$$
n \lambda R_{1 \overline{1}}-R R_{1 \overline{1}}=\lambda R-|R c|^{2}-\frac{n-1}{n+1} R_{1 \overline{1}}|\nabla f|^{2}
$$

and

$$
(n+1) \lambda R_{1 \overline{1}}-R R_{1 \overline{1}}-R_{1 \overline{1}}^{2}=-\frac{1}{n+1} R_{1 \overline{1}}|\nabla f|^{2} .
$$

Proof. In (2.6), setting $i=j=1$, we have

$$
\begin{aligned}
& \lambda R_{1 \overline{1}}-\frac{1}{n+1} R_{1 \overline{1}}^{2}-\frac{1}{n+1} R R_{1 \overline{1}} \\
& =\lambda R_{1 \overline{1}}-\frac{2}{n+1} R_{1 \overline{1}}^{2}-\frac{1}{n+1} R_{1 \overline{1}}\left(R-R_{1 \overline{1}}\right) \\
& =\lambda R_{1 \overline{1}}-\frac{2}{n+1} R_{1 \overline{1}}^{2}-\frac{1}{n+1} R_{1 \overline{1}} \sum_{k=2}^{n} R_{k \bar{k}} \\
& =\lambda R_{1 \overline{1}}-R_{1 \overline{1} 1 \overline{1}} R_{1 \overline{1}}-\sum_{k=2}^{n} R_{1 \overline{1} k \bar{k}} R_{k \bar{k}} \\
& =\lambda R_{1 \overline{1}}-\sum_{k=1}^{n} R_{1 \overline{1} k \bar{k}} R_{k \bar{k}} \\
& =\frac{1}{n+1}\left[\frac{1}{n+1} R_{1 \overline{1}}|\nabla f|^{2}+\lambda R-|R c|^{2}-\frac{n}{n+1} R_{1 \overline{1}}|\nabla f|^{2}+\lambda R_{1 \overline{1}}-R_{1 \overline{1}}^{2}\right] .
\end{aligned}
$$

Thus, formula (2.9) follows immediately.

Next, by setting $i=1$ in (2.7) and dividing both sides of the equation by $\nabla_{1} f$, we get (2.10).

Proposition 2.5. At a point $p$ where $\nabla f \neq 0$, we have either

$$
R c(\nabla f, \nabla f)=0
$$

or

$$
R c(\nabla f, \nabla f)=\frac{\lambda}{n+4}|\nabla f|^{2} .
$$

Proof. Since at point $p, \nabla f \neq 0$, formula (2.10) implies that in a neighborhood of $p$ we have

$$
\left[(n+1) \lambda-R-\frac{R_{j \bar{i}} \nabla_{i} f \nabla_{\bar{j}} f}{|\nabla f|^{2}}+\frac{1}{n+1}|\nabla f|^{2}\right] \frac{R_{j \bar{i}} \nabla_{i} f \nabla_{\bar{j}} f}{|\nabla f|^{2}}=0 .
$$


Therefore, there are two possibilities:

I) $R_{j \bar{i}} \nabla_{i} f \nabla_{\bar{j}} f=0$ at $p$

or

II) $R_{j i} \nabla_{i} f \nabla_{\bar{j}} f \neq 0$ at $p$. In this case, near $p$ we have

$$
-(n+1) \lambda+R+\frac{R_{j \bar{i}} \nabla_{i} f \nabla_{\bar{j}} f}{|\nabla f|^{2}}-\frac{1}{n+1}|\nabla f|^{2}=0 .
$$

Taking the covariant derivative on both sides gives us

$$
\begin{aligned}
0 & =\nabla_{k} R+\frac{1}{|\nabla f|^{2}}\left(\nabla_{i} f \nabla_{\bar{j}} f \nabla_{k} R_{j \bar{i}}+R_{j \bar{i}} \nabla_{i} f \nabla_{k} \nabla_{\bar{j}} f\right)-\frac{\nabla_{j} f \nabla_{k} \nabla_{\bar{j}} f}{|\nabla f|^{4}} R_{l_{\bar{i}}} \nabla_{i} f \nabla_{\bar{l}} f \\
& -\frac{1}{n+1}\left(\nabla_{j} f \nabla_{k} \nabla_{\bar{j}} f\right) \\
& =\nabla_{k} R+\frac{1}{(n+1)|\nabla f|^{2}} \nabla_{i} f \nabla_{\bar{j}} f\left(\nabla_{k} R g_{j \bar{i}}+\nabla_{j} R g_{k \bar{i}}\right)+\frac{1}{|\nabla f|^{2}}\left(\lambda \nabla_{k} R-R_{k \bar{j}} \nabla_{j} R\right) \\
& -\frac{\lambda \nabla_{k} f-\nabla_{k} R}{|\nabla f|^{4}} \nabla_{i} R \nabla_{\bar{i}} f-\frac{1}{n+1}\left(\lambda \nabla_{k} f-\nabla_{k} R\right) .
\end{aligned}
$$

Evaluating the identity above at $p$ under the orthonormal frame $\left\{w_{1}, w_{2}, \cdots, w_{n}\right\}$ yields

$$
\begin{aligned}
0 & =R_{1 \overline{1}}+\frac{2}{(n+1)|\nabla f|^{2}} R_{1 \overline{1}}|\nabla f|^{2}+\frac{1}{|\nabla f|^{2}}\left(\lambda R_{1 \overline{1}}-R_{1 \overline{1}}^{2}\right) \\
& \quad-\frac{\lambda-R_{1 \overline{1}}}{|\nabla f|^{4}} R_{1 \overline{1}}|\nabla f|^{2}-\frac{1}{n+1}\left(\lambda-R_{1 \overline{1}}\right) \\
= & \frac{n+4}{n+1} R_{1 \overline{1}}-\frac{1}{n+1} \lambda .
\end{aligned}
$$

Thus, we have $R c(\nabla f, \nabla f)=\frac{\lambda}{n+4}|\nabla f|^{2}$ whenever $R c(\nabla f, \nabla f) \neq 0$.

Now we are ready to prove the main theorems.

First, we may assume that $f \neq C$ Const, for otherwise we get that $M$ is KählerEinstein from the soliton equation.

Proof of Theorem 1.3. For steady Kähler-Ricci solitons, we have $\lambda=0$.

From Proposition 2.2, we know that $R c(\nabla f, \nabla f)=\frac{\lambda}{n+4}|\nabla f|^{2}=0$ whenever $R c(\nabla f, \nabla f) \neq 0$, which is a contradiction. Therefore, we always have $R c(\nabla f, \nabla f)=$ 0 . Then (2.9) implies that $R c=0$ in the set $\{p \in M \mid \nabla f(p) \neq 0\}$. On the other hand, by the soliton equation, it is easy to see that we also have $R c=0$ in the interior of the set $\{p \in M \mid \nabla f(p)=0\}$. Thus the steady soliton $M$ must be Kähler-Ricci flat.

Proof of Theorem 1.4. For shrinking and expanding Kähler-Ricci solitons, we have $\lambda \neq 0$.

In this case, from Proposition 2.2 and the continuity of $\frac{R c(\nabla f, \nabla f)}{|\nabla f|^{2}}$, we conclude that in each component of the open set $A=\{p \in M \mid \nabla f(p) \neq 0\}$, we have either $R c(\nabla f, \nabla f)=\frac{\lambda}{n+4}|\nabla f|^{2}$ or $R c(\nabla f, \nabla f)=0$.

If $R c(\nabla f, \nabla f)=\frac{\lambda}{n+4}|\nabla f|^{2}$ in some component $\Omega$ of $A$, then at any point $p \in \Omega$ we have $R_{1 \overline{1}}=\frac{\lambda}{n+4}$ and $\nabla R(p)=\frac{\lambda}{n+4} \nabla f(p)$ from formula (2.4). Therefore, we have $\nabla R=\frac{\lambda}{n+4} \nabla f$ in $\Omega$. It then follows that $R=\frac{\lambda}{n+4} f+C$ in $\Omega$. Thus (2.10) 
implies that $|\nabla f|^{2}=\frac{n+1}{n+4} \lambda f+C$ in $\Omega$. Since $R+|\nabla f|^{2}-\lambda f=C_{0}$, we have $f=C_{1}$ in $\Omega$, which contradicts the fact that $\nabla f \neq 0$ in $\Omega$.

Therefore, we must have $R c(\nabla f, \nabla f)=0$ in $A$. Since $f=C o n s t$ in the interior of $M \backslash A$, we have $R c(\nabla f, \nabla f)=0$ on the whole manifold $M$. It follows that $\nabla R=0$ on $M$. Then (2.5) implies that the Ricci curvature tensor is parallel on $M$. Therefore, by the de-Rahm decomposition theorem, the universal cover of $M$ is isometric to $N^{n-1} \times \mathbb{C}$, where $N$ is again an $(n-1)$-dimensional Kähler-Ricci soliton with harmonic Bochner tensor. Thus by induction, we can finally get that $M$ is isometric to a quotient of the product of a Kähler-Einstein manifold and the complex Euclidean space.

This finishes the proof.

\section{ACKNOWLEDGMENTS}

The authors would like to thank their advisor, Professor Huai-Dong Cao, for suggesting this problem and for stimulating discussions. We are grateful for his constant encouragement and support. We would also like to thank the referee for many helpful suggestions and comments.

\section{REFERENCES}

1. Brendle, S., Uniqueness of gradient Ricci solitons, Math. Res. Lett. 18 (2011), no. 3, 531-538. MR2802586

2. Bryant, R., Bochner-Kähler metrics, J. Amer. Math. Soc. 14 (2001), no. 3, 623-715. MR.1824987 (2002i:53096)

3. Bryant, R., Gradient Kähler Ricci solitons, Géométrie différentielle, physique mathématique, mathématiques et société. I. Astérisque No. 321 (2008), 51-97. MR2521644(2010i:53138)

4. Cao, H.-D., Recent progress on Ricci solitons, Recent advances in geometric analysis, 1-38, Adv. Lect. Math. (ALM), 11, Int. Press, Somerville, MA, 2010. MR2648937 (2011d:53061)

5. Cao, H.-D., Geometry of complete gradient shrinking Ricci solitons, Geometric Analysis (Vol. I), 227-246, Adv. Lect. Math. (ALM), 17, Int. Press, Somerville, MA, 2011.

6. Cao, H.-D., Catino, G., Chen, Q., Mantegazza, C. and Mazzieri, L., Bach-flat gradient steady Ricci solitons, arXiv:1107.4591

7. Cao, H.-D., Chen, B.-L. and Zhu, X.-P., Recent developments on Hamilton's Ricci flow, Surveys in differential geometry. Vol. XII, Int. Press, Somerville, MA, 2008. MR2488948 (2010c:53096)

8. Cao, H.-D. and Chen, Q., On Locally Conformally Flat Gradient Steady Ricci Solitons, Trans. Amer. Math. Soc. 364 (2012), 2377-2391.

9. Cao, H.-D. and Chen, Q., On Bach-flat gradient shrinking Ricci solitons, arXiv:1105.3163

10. Cao, H.-D. and Hamilton, R., Gradient Kähler-Ricci solitons and periodic orbits, Comm. Anal. Geom. 8 (2000), no. 3, 517-529. MR1775136 (2001g:53117)

11. Cao, H.-D. and Zhu, X.-P., A complete proof of the Poincaré and geometrization conjecturesapplication of the Hamilton-Perelman theory of the Ricci flow, Asian J. Math. 10 (2006), no. 2, 165-492. MR2233789 (2008d:53090)

12. Cao, X., Wang, B. and Zhang, Z., On locally conformally flat gradient shrinking Ricci solitons, Commun. Contemp. Math. 13 (2011), no. 2, 269-282. MR2794486

13. Catino, G. and Mantegazza, C., The evolution of the Weyl tensor under the Ricci flow, Ann. Inst. Fourier 6 (2011), no. 4, 1407-1435.

14. Chau, A. and Tam, L.-F., A note on the uniformization of gradient Kähler Ricci solitons, Math. Res. Lett. 12 (2005), no. 1, 19-21. MR2122726 (2005i:53081)

15. Chen, X.X. and Wang, Y., On four-dimensional anti-self-dual gradient Ricci solitons, arXiv:1102.0358

16. Eminenti, M., La Nave, G. and Mantegazza. C., Ricci solitons: the equation point of view, Manuscripta Math. 127 (2008), 345-367. MR2448435 (2009m:53088)

17. Fernández-López, M. and García-Río, E. Rigidity of shrinking Ricci solitons, Math. Z. 269 (2010), no. 1-2, 461-466. MR2836079 
18. Hamilton, R., The Ricci flow on surfaces, Contemp. Math., 71, Amer. Math. Soc., Providence, RI, 1988, 237-262. MR954419 (89i:53029)

19. Ivey, T., Ricci solitons on compact three-manifolds, Diff. Geom. Appl. 3 (1993), 301-307. MR $1249376(94 \mathrm{j}: 53048)$

20. Munteanu, O. and Sesum, N., On gradient Ricci solitons, arXiv:0910.1105, to appear in J. Geom. Anal.

21. Naber, A., Noncompact shrinking four solitons with nonnegative curvature, J. Reine Angew. Math. 645 (2010), 125-153. MR2673425

22. Ni, L. and Wallach, N., On a classification of gradient shrinking solitons, Math. Res. Lett. 15 (2008), no. 5, 941-955. MR2443993 (2010i:53127)

23. Perelman, G., Ricci flow with surgery on three manifolds, arXiv:math.DG/0303109.

24. Petersen, P. and Wylie, W., Rigidity of gradient Ricci solitons, Pacific J. Math. 241 (2009), no. 2, 329-345. MR2507581 (2010j:53071)

25. Petersen, P. and Wylie, P., On the classification of gradient Ricci solitons, Geom. Topol. 14 (2010), no. 4, 2277-2300. MR2740647 (2012a:53060)

26. Su, Y. and Zhang, K., On the Kähler-Ricci solitons with vanishing Bochner-Weyl tensor, preprint.

27. Zhang, Z.-H., Gradient shrinking solitons with vanishing Weyl tensor, Pacific J. Math. 242 (2009), no. 1, 189-200. MR2525510 (2010f:53116)

Department of Mathematics, Lehigh University, Bethlehem, Pennsylvania 18015

E-mail address: qic208@lehigh.edu

Department of Mathematics, Lehigh University, Bethlehem, Pennsylvania 18015

E-mail address: mez206@lehigh.edu 